\title{
Energy-loss contribution to grazing scattering of fast He atoms from a silver surface
}

\author{
C. A. Ríos Rubiano, ${ }^{1}$ G. A. Bocan, ${ }^{2}$ J. I. Juaristi, ${ }^{3,4}$ and M. S. Gravielle ${ }^{1, *}$ \\ ${ }_{1}^{1}$ Instituto de Astronomía y Física del Espacio (CONICET-UBA), Casilla de Correo 67, Sucursal 28, 1428 Buenos Aires, Argentina \\ ${ }^{2}$ Centro Atómico Bariloche, Comisión Nacional de Energía Atómica, and Consejo Nacional de Investigaciones Científicas y Técnicas, \\ S.C. de Bariloche, Río Negro, Argentina \\ ${ }^{3}$ Departamento de Física de Materiales, Facultad de Químicas, UPV/EHU, 20018 San Sebastián, Spain \\ ${ }^{4}$ Donostia International Physics Center (DIPC) and Centro de Física de Materiales CFM/MPC (CSIC-UPV/EHU), \\ 20018 San Sebastián, Spain
}

(Received 20 December 2013; published 12 March 2014)

\begin{abstract}
The energy lost by helium atoms axially scattered from a $\mathrm{Ag}(110)$ surface is studied in order to investigate the influence of dissipative processes on fast atom diffraction spectra. In this work inelastic projectile distributions are evaluated within a semiclassical formalism that includes dissipative effects due to electron-hole excitations through a friction force. For incidence along the [1 12$]$ and [1ㅣㅣ directions the model predicts the presence of multiple peaks in the energy-loss spectrum for a given impact energy. But these structures are completely washed out when the experimental dispersion of the incident beam is taken into account, giving rise to a smooth energy-loss distribution. Simulations including the experimental energy spread are in fairly good agreement with available experimental data for the [11̄2] channel. In addition, our results suggest that inelastic processes produce an almost constant background in the transverse momentum distribution, except in the extremes of the momentum range where classical rainbow maxima appear. By adding elastic and inelastic contributions, experimental diffraction patterns are well reproduced.
\end{abstract}

DOI: 10.1103/PhysRevA.89.032706

PACS number(s): $34.35 .+\mathrm{a}, 34.50 . \mathrm{Bw}, 68.49 . \mathrm{Bc}$

\section{INTRODUCTION}

Since the unexpected observation of grazing incidence diffraction of fast atoms (GIFAD) on crystal surfaces [1,2], extensive research, both experimental and theoretical, has been devoted to the subject [3-11]. The first experimental evidences of this phenomenon were reported at insulator materials [1,2], where the presence of a wide band gap helps to suppress inelastic processes, thus preventing quantum decoherence [12]. Soon afterwards the effect was observed at semiconductor [13] and metallic surfaces [7,14], even though in the last case, energy-loss values were found to be significant $[7,15,16]$. The observation of GIFAD for metals was rather unforeseen as no threshold is present for electronic excitations and consequently, inelastic electronic processes were expected to play an important role against coherence. Also meaningful energy losses were recently reported for grazing scattering of $\mathrm{H}$ atoms from a $\mathrm{LiF}(001)$ surface [12,17], where inelastic electronic processes were found to produce a diffuse background in the experimental GIFAD distributions.

The aim of this work is to study the energy lost by swift He atoms after grazing impinging on a $\mathrm{Ag}(110)$ surface along lowindexed crystallographic directions, under the same conditions for which diffraction has been observed [7]. This collision system corresponds to the first and simplest metallic case for which experimental GIFAD distributions were reported in conjunction with measurable energy losses [7].

In a previous paper [18], GIFAD patterns for He atoms scattered off $\operatorname{Ag}(110)$ were investigated considering different energies associated with the motion normal to the surface plane. The calculations were performed to represent the elastic

\footnotetext{
*Author to whom correspondence should be addressed: msilvia@iafe.uba.ar
}

regime, neglecting inelastic contributions and associated decoherence. The elastic collision process was described within the surface eikonal (SE) approximation [6], while inherent and experimental uncertainties were empirically taken into account by convoluting the calculated diffraction intensities with the observed Lorentzian profile. The SE approach is a semiclassical method that includes the quantum interference among contributions coming from different projectile paths, which are classically evaluated varying the initial position $[6,19]$. Here we study the projectile energy loss associated with electronic transitions from the metal surface by means of a semiclassical formalism. It takes into account the energy lost by the atom along the classical trajectory but without including effects of quantum coherence. The influence of quantum interferences in the projectile energy-loss spectrum is expected to be minor because, even for insulator surfaces, where valence electrons are tighter than for metals, coherence quantum effects are completely washed out when partial contributions coming from different initial crystal states are added to obtain the transition probability to a given final state [20].

The energy loss for the $\mathrm{He} / \mathrm{Ag}(110)$ system has previously been addressed by Refs. [15,16]. In their model the electron-helium elastic cross sections were taken from gas phase experiments [21], while the electron density function was calculated within the jellium model, assuming planar symmetry. The associated decoherence was estimated in the form of a momentum broadening perpendicular to the beam direction, i.e., in the plane where diffraction is observed.

In this article our approach is different. We use a detailed description of the surface, including its full three-dimensional (3D) electron density profile, to evaluate both the elastic diffraction, considered as fully coherent, and the inelastic scattering, assumed as fully incoherent. The final projectile distribution is then interpreted as a linear combination of these two contributions. While the elastic scattering is described 
within the SE approximation [18], the inelastic contribution is derived by introducing a friction force in Newton's equations for the projectile trajectory. The friction force is expressed in terms of the transport cross section at the Fermi level, corresponding to the screened potential of the atom embedded in an electron gas with electronic density equal to that of the surface at the position of the atom [22]. Both the surface potential and the electronic density are evaluated on equal footing, i.e., from density-functional theory (DFT) calculations within the same conditions. The projectile-surface interaction is represented by a potential energy surface (PES) that was built from a large set of ab initio data obtained with the DFT-based QUANTUM ESPRESSO code [23], combined with a sophisticated interpolation technique [24]. The quality of such a PES was tested by means of GIFAD patterns for perpendicular energies in the range $0.1 \mathrm{eV}$ to $0.5 \mathrm{eV}$ in Ref. [18]. Note that no average of the surface potential nor the electronic density along the incidence direction was considered in the calculation. Such an average was shown to be a good simplification for the elastic regime [2,5,10,25]; however its validity to describe electronic transitions is not established.

Angle and energy-loss distributions of $\mathrm{He}$ atoms inelas-

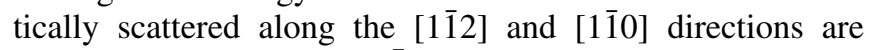
investigated. Since the [110] channel presents the largest corrugation of the surface potential across it, as observed both in the experiment [15] and in the calculations [18], we center on this direction our analysis of the contribution of inelastic processes to experimental diffraction spectra. The paper is organized as follows. The theoretical model is summarized in Sec. II, results are presented and discussed in Sec. III, and in Sec. IV we outline our conclusions. Atomic units (a.u.) are used unless otherwise stated.

\section{THEORETICAL MODEL}

The final projectile distribution originated by inelastic collisions with the surface is derived from classical trajectory calculations by including the energy lost by the atom along the classical path. The energy loss suffered by the projectile is calculated from the so-called electronic stopping power, which is the energy lost per unit path length or, in other words, the dissipative force experienced by the moving atom. For grazing incidence with energies in the $\mathrm{keV}$ range, electron-hole pair excitations represent the main mechanism of projectile energy loss, while contributions due to nuclear scattering are expected to be negligible because the projectile travels far away from the topmost atomic plane [26].

The stopping power of atoms moving through metals with velocities lower than the Fermi velocity of the metal has a linear dependence on velocity [27]. This reflects the fact that in a metal there is no minimum energy required to excite electron-hole pairs. Then, it allows one to treat the energy loss suffered by the projectile by introducing a friction force in the classical equations of motion [28]. Here, the friction force for helium atoms interacting with a silver surface is calculated within the local density friction approximation (LDFA) [22]. In contrast to earlier energy-loss calculations [29], this is a parameter-free model that incorporates energy exchange with the surface into the dynamics while keeping the accuracy of the three-dimensional $a b$ initio PES for the projectile-surface interaction. The LDFA has been successfully applied to study dissipative effects for atoms and molecules interacting with different metal surfaces, including the reactive $\mathrm{N} / \mathrm{Ag}(111)$ system [30-32].

Briefly, within the LDFA one calculates the electronic density $n(\vec{R})$ at each position $\vec{R}$ along the classical trajectory from abinitio calculations and within the same conditions as the PES. The friction coefficient at each point of the trajectory $\mu(\vec{R})$ is, subsequently, approximated by that corresponding to an electron gas with electronic density $n_{0}=n(\vec{R})$. This friction coefficient is calculated in terms of the exact transport cross section at the Fermi level for electrons scattered at the potential induced by the $\mathrm{He}$ projectile in the electron gas [33]. This potential is evaluated using DFT for a helium atom embedded in an electron gas [34]. In this way, the model includes nonlinear effects both in the medium response to the atomic potential (nonlinear screening) and in the calculation of the relevant cross sections for the energy-loss process.

Finally, the classical equation of motion that one has to solve reads [22,30-32]

$$
m_{P} \frac{d^{2} \vec{R}}{d t^{2}}=-\vec{\nabla} V_{S P}(\vec{R})-\mu(\vec{R}) \frac{d \vec{R}}{d t},
$$

where $\vec{R}$ is the projectile position vector, $m_{P}$ is the projectile mass, and $V_{S P}(\vec{R})$ is the projectile-surface potential. The first term on the right side of Eq. (1) is the adiabatic force obtained from the 3D PES, while the second term is the dissipative force experienced by the atom.

The final momentum distribution of inelastically scattered atoms, $d P^{\text {(inel) }} / d E_{f} d \Omega_{f}$, is obtained by counting the number of classical trajectories ending with final momentum $\vec{K}_{f}$ in the direction of the solid angle $\Omega_{f} \equiv\left(\theta_{f}, \varphi_{f}\right)$ and with final energy $E_{f}=K_{f}^{2} /\left(2 m_{P}\right)$, where $\theta_{f}$ and $\varphi_{f}$ are the final polar and azimuthal angles, respectively, with $\varphi_{f}$ measured with respect to the incidence direction in the surface plane.

\section{Projectile-surface interaction}

The interaction energy of the He atom with the $\operatorname{Ag}(110)$ surface is described with a full adiabatic 3D PES that depends on the atomic position $\vec{R}=(X, Y, Z)$, where $Z$ represents the distance to the topmost atomic plane. The PES is constructed from a grid of $a b$ initio energies for $42 Z$ values and 6 $(X Y)$ sites on the surface plane, over which an interpolation is performed [24]. Details of PES calculations [18] are here summarized.

All $a b$ initio data are obtained from the DFT-based QUANTUM ESPRESSO code [23]. The values of relevant input parameters are chosen so that $a b$ initio energies are calculated to a prescribed accuracy (differences $<5 \mathrm{meV}$ with respect to the converged result). The exchange-correlation energy is calculated within the generalized gradient approximation (GGA), using the Perdew-Burke-Ernzerhof energy functional (PBE) [35]. The electron-core interaction is described with ultrasoft pseudopotentials [36]. The energy cutoff in the planewave expansion is $0.48 \mathrm{keV}$ for the wave functions and $3.3 \mathrm{keV}$ for the charge density and potential; the fractional occupancies are determined through the broadening approach of MarzariVanderbilt [37] with $\sigma=0.14 \mathrm{eV}$; and the Brillouin-zone 
integration is performed with a $10 \times 7 \times 1$ Monkhorst-Pack grid of special $k$ points. The $\mathrm{Ag}$ lattice constant is $a=$ 7.865 a.u.

The $\mathrm{Ag}(110)$ surface is modeled by means of the supercellslab scheme. A four-layer slab is used with a $2 \times 2$ cell in the plane parallel to the surface and a supercell of length 25.03 a.u. along the normal to the surface ( $\hat{z}$ axis). The energy for $\mathrm{He}-\mathrm{Ag}(110)$ with the He atom midway between slabs provides a reasonable representation of the asymptotic region and is chosen as the energy reference. The surface interlayer distance is relaxed from its bulk value $d_{0}=2.781$ a.u., keeping the two bottom layers fixed. Geometry corrections due to relaxation amount to $-9.14 \%$ and $+4.11 \%$ for the first and second interlayer distances, respectively, in accord with experimental results [38]. Once relaxed, the slab is kept frozen for the calculations that follow.

Given the closed-shell electronic structure of the He atom $\left(1 s^{2}\right)$, we perform a non-spin-polarized calculation of the ground state. A quality check of the interpolation shows that the error introduced is $<1 \mathrm{meV}$, well below the prescribed accuracy for the $a b$ initio data.

\section{RESULTS}

In this work ${ }^{3} \mathrm{He}$ atoms grazingly colliding with $\mathrm{Ag}(110)$ along two different crystallographic directions-[112] and [110] — are studied considering incidence energies in the few keV range. Energy- and angle-resolved distributions of inelastically scattered atoms are classically derived by solving Eq. (1) for different initial positions. In all the cases $4 \times 10^{5}$ trajectories with random initial positions that vary within a surface area equal to $4 \times 4$ unit cells are used. For all the trajectories the initial distance of the atom from the surface is chosen equal to the lattice constant $a$, corresponding to a region where the surface interaction is completely negligible. The differential probability $d P^{(\mathrm{inel})} / d E_{f} d \Omega_{f}$ is calculated by considering a dense grid of $E_{f}, \theta_{f}$, and $\varphi_{f}$ values $(100 \times 100 \times 100)$, which is used to build the cells where final momenta $\vec{K}_{f}$ are assigned. The energy-loss distribution $d P^{(\text {inel })} / d \omega$, as a function of the lost energy $\omega=E_{i}-$ $E_{f}$, with $E_{i}$ the initial energy, is straightforwardly derived from $d P^{(\text {inel })} / d E_{f} d \Omega_{f}$ by integrating on the solid angle $\Omega_{f}$. Figure 1(a) shows the friction coefficient $\mu$ for the He atom moving in an electron gas of local density $n_{0}$, as a function of the mean electron radius $r_{s}$, defined as $r_{s}=\left[3 /\left(4 \pi n_{0}\right)\right]^{1 / 3}$. Equipotential curves along the considered incidence directions, averaged across the channel, are plotted in Fig. 1(b).

First, in order to verify the validity of the theoretical method for the $\mathrm{He}-\mathrm{Ag}(110)$ system, we consider incidence along the [11 2 ] channel for which experimental energy-loss data were reported in Refs. [16,39]. Simulated and experimental energy-loss spectra for the impact energy $E_{i}=0.5 \mathrm{keV}$ are displayed in Fig. 2. In the figure we show theoretical results corresponding to a monoenergetic incident beam, together with values obtained by including the experimental energy spread by means of a convolution [40]. We found that when the initial energy dispersion is not considered in the calculation, the theoretical energy-loss distribution, named here the primary distribution (blue dashed line), presents a double-peaked structure with maxima at the lowest and highest (a)
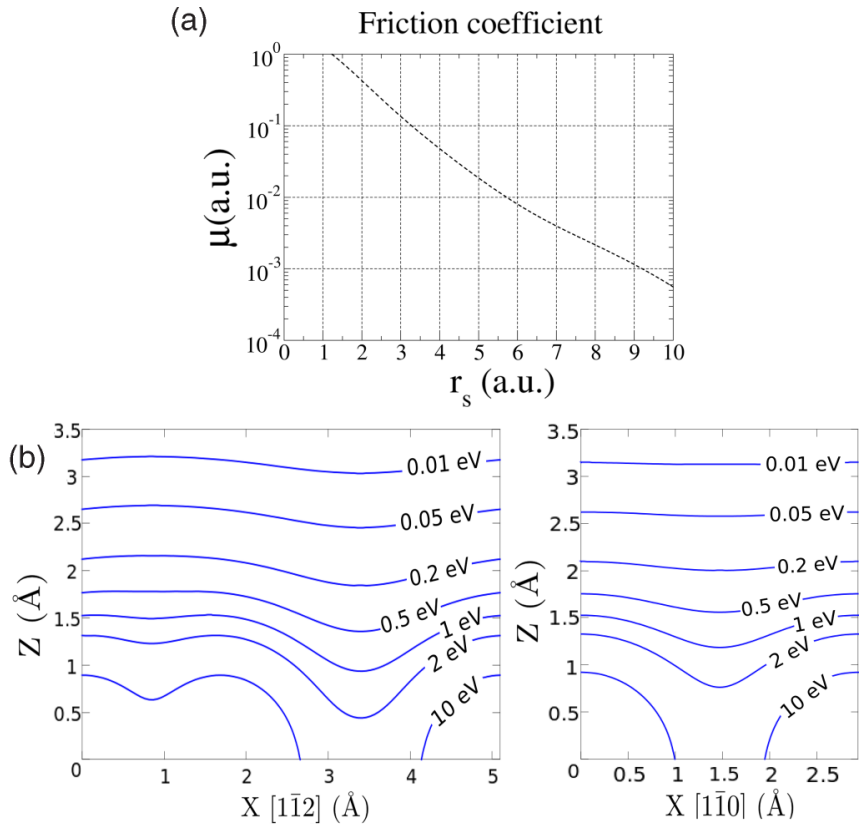

FIG. 1. (Color online) (a) Friction coefficient for He in an electron gas as a function of the mean electron radius $r_{s}$. (b) Distances $Z$ to the surface plane (topmost atomic layer) of equipotential curves for the interaction between the He atom and the $\operatorname{Ag}(110)$ surface, averaged across the channel, as a function of the coordinates along the [1 12$]$ and [1 10$]$ directions, respectively. In both cases, the value $X=0$ corresponds to a site on a topmost Ag atom.

lost-energy values, respectively (see the inset for more detail). These sharp peaks, labeled as P1 and P2 in the inset, are associated with projectiles that move in the middle or over the rows of atoms forming the channel, probing regions with low or high electronic densities, respectively, as observed in Fig. 3. Even though evidences of trajectory-dependent energy-loss

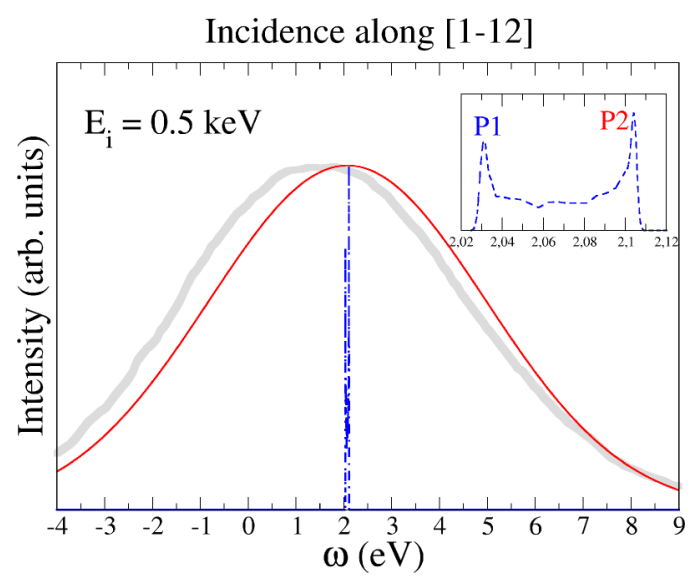

FIG. 2. (Color online) Energy-loss spectrum, as a function of the lost energy $\omega$, for ${ }^{3} \mathrm{He}$ atoms impinging on a $\mathrm{Ag}(110)$ surface along the [11 2 ] direction. The incidence energy and angle are $E_{i}=0.5 \mathrm{keV}$ and $\theta_{i}=1.5^{\circ}$, respectively. Blue dashed line, differential probability $d P^{\text {(inel) }} / d \omega$ for a monoenergetic incident beam (primary spectrum); red solid line, differential probability convoluted to include the experimental energy spread; gray solid line, experimental data from Refs. [16,39]. Inset: Detail of the primary spectrum. 
Incidence along [1-12]

(a)

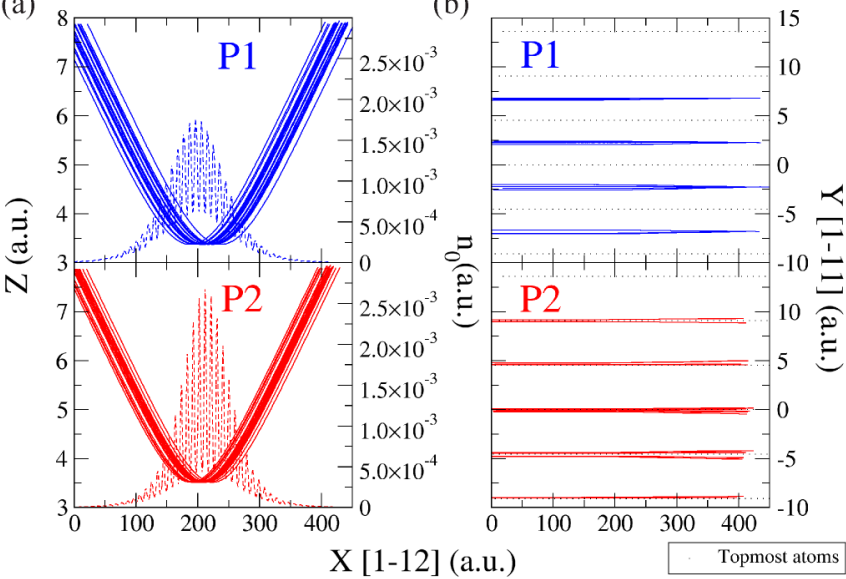

FIG. 3. (Color online) For the case of Fig. 2, representative trajectories contributing to the peaks P1 and P2 of the inset of Fig. 2. (a) Distance $Z$ to the topmost atomic layer (solid line) and electron density $n_{0}$ (dashed line) along the trajectory, as a function of the coordinate $X[1 \overline{1} 2]$ along the incidence direction. (b) Similar to (a) for the transversal position $Y[1 \overline{1} 1]$ with respect to the incidence direction $X$. Dots, positions of topmost $\mathrm{Ag}$ atoms.

structures have indeed been observed for axial channeling collisions where projectile penetration into the atomic surface can occur [28,41], in the present case the energy-loss structures of the primary spectrum are completely washed out when the experimental resolution for the initial conditions is taken into account. The convoluted energy-loss distribution shows a smooth behavior, with a broad maximum whose position is in fairly good agreement with the experimental one. Note that the LDFA is based on a local electronic density whose use might be questionable if the electronic density changes appreciably. At long distances from the surface, such as the ones involved here, the surface electronic density decreases steeply as the distance to the surface increases. In addition, the [112] channel presents a low corrugation of the potential across it, as extracted from GIFAD patterns [18], but a strong corrugation along the channel, as observed in Fig. 1(b). This last electronic density variation is the one probed by the energy-loss spectrum. Then, taking into account the sensitivity of mean energy loss values to the models used to describe both the friction and the surface interaction [28], the reasonable agreement observed in Fig. 2 is an indication of the applicability of the method for grazing incidence conditions.

To investigate the contribution of inelastic processes to GIFAD patterns, hereafter we will concentrate on scattering along the [1ㅣㅣㄹ direction, for which rich GIFAD patterns were observed [18] due to the strong corrugation of the potential across the channel. In Fig. 4 inelastic differential probabilities $d P^{\text {(inel) }} / d \omega$ for incidence along the [1 $\left.1 \overline{1} 0\right]$ channel with energy $E_{i}=1 \mathrm{keV}$ are plotted as a function of $\omega$. Results for a monoenergetic incident beam are displayed again with blue dashed line. This primary distribution presents three welldefined peaks, labeled as $\mathrm{P}^{\prime}$, $\mathrm{P} 2^{\prime}$, and $\mathrm{P}^{\prime}$, which resemble the energy-loss rainbow structures reported in Ref. [42]. In order to understand the origin of these energy-loss structures, in Fig. 5

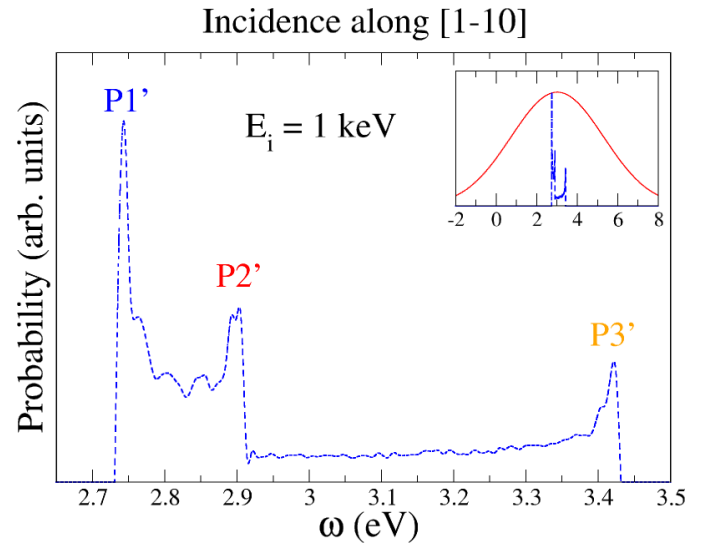

FIG. 4. (Color online) Energy-loss spectrum, as a function of the lost energy $\omega$, for ${ }^{3} \mathrm{He}$ atoms impinging on a $\operatorname{Ag}(110)$ surface along the [110] direction. The incidence energy and angle are $E_{i}=1 \mathrm{keV}$ and $\theta_{i}=1^{\circ}$, respectively. Blue dashed line, differential probability $d P^{(\text {inel) }} / d \omega$ for a monoenergetic incident beam (primary spectrum). Inset: Red solid line, differential probability convoluted to include the experimental energy spread.

we plot representative trajectories that contribute to each peak. The peak $\mathrm{P}^{\prime}$, which presents the highest intensity, is associated with trajectories that suffer strong azimuthal deviations with respect to the incidence direction, corresponding to classical rainbow scattering (see Fig. 7 below). Such paths probe regions with low electron density, producing the lowest energy loss of the primary spectrum. Instead, the trajectories that contribute to the peak P3' correspond to He atoms that move over the rows of topmost $\mathrm{Ag}$ atoms that form the channel, suffering almost no deviation. Even though these projectiles are the ones that approach less to the surface because they do not enter into the channel, they probe the region with the highest electron

\section{Incidence along [1-10]}

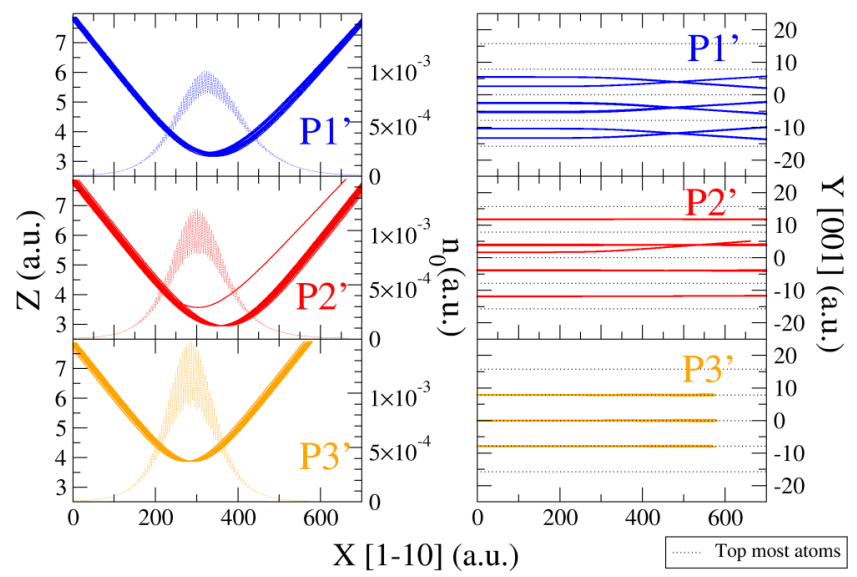

FIG. 5. (Color online) For the case of Fig. 4, representative trajectories contributing to the peaks $\mathrm{P} 1^{\prime}, \mathrm{P} 2^{\prime}$, and $\mathrm{P} 3^{\prime}$ of Fig. 4. (a) Distance $Z$ to the topmost atomic layer (solid line) and electron density $n_{0}$ (dashed line) along the trajectory, as a function of the coordinate $X$ along the [110] direction. (b) Similar to (a) for the transversal position $Y[001]$ with respect to the incidence direction $X$. Dots, positions of topmost Ag atoms. 
density, thus suffering the highest energy loss. In turn, two different kinds of trajectories contribute to the peak $\mathrm{P}^{\prime}$ : one of them running parallel to the channel in the middle position between rows and the other running far away from the surface plane and suffering an azimuthal deflection.

From the analysis of the primary spectra of Figs. 2 and 4 we conclude that energy-loss peaks might provide detailed information about the trajectory-dependent energy loss and, consequently, about the atom-surface interaction. However, a resolution better than $1 \mathrm{eV}$ would be required to experimentally identify such energy-loss structures. In Fig. 4, when the initial experimental conditions are taken into account by convoluting the primary distribution with the energy profile of the incident beam [43], a smooth energy-loss curve is obtained, as shown in the inset. The same spectrum was also derived by introducing in our calculations an initial $E_{i}$ distribution reproducing the experimental incident beam profile. It proves that the shape of the primary energy-loss distribution is not affected by the typical experimental variation of the impact energy. The mean energy loss of the simulated spectrum is around $3 \mathrm{eV}$, which compares with the value reported in Ref. [7] for a different direction [44]. The same holds for $E_{i}=0.5 \mathrm{keV}$ and $E_{i}=$ $2 \mathrm{keV}$, shown in Figs. 6(a) and 6(b), where mean energy-loss values are around $0.6 \mathrm{eV}$ and $13 \mathrm{eV}$, respectively. Besides, from the comparison of Figs. 4 and 6 we found that the relative positions of the energy-loss peaks- $-\mathrm{P}^{\prime}, \mathrm{P}^{\prime}$, and $\mathrm{P} 3^{\prime}$ - vary with the impact energy. Peak P2' moves from low to high $\omega$ values as $E_{i}$ increases.

We also analyze the angular distribution of inelastically scattered He atoms. As in the case of elastic scattering [6,45], we found that the initial and final energies associated with the motion normal to the channel are very similar; that is, final
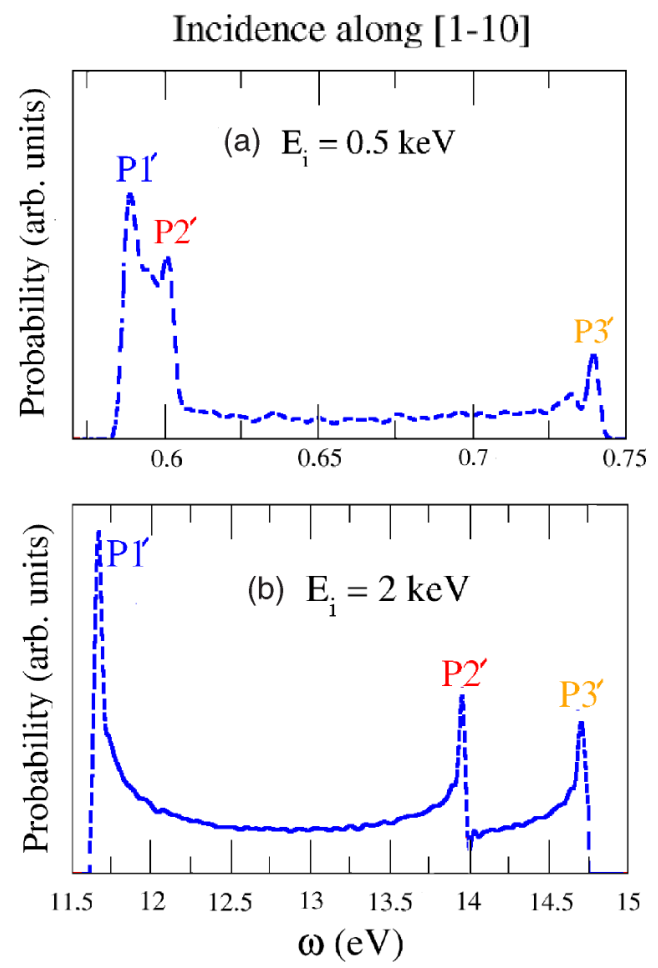

FIG. 6. (Color online) Similar to Fig. 4 for (a) $E_{i}=0.5 \mathrm{keV}$ and (b) $E_{i}=2 \mathrm{keV}$.

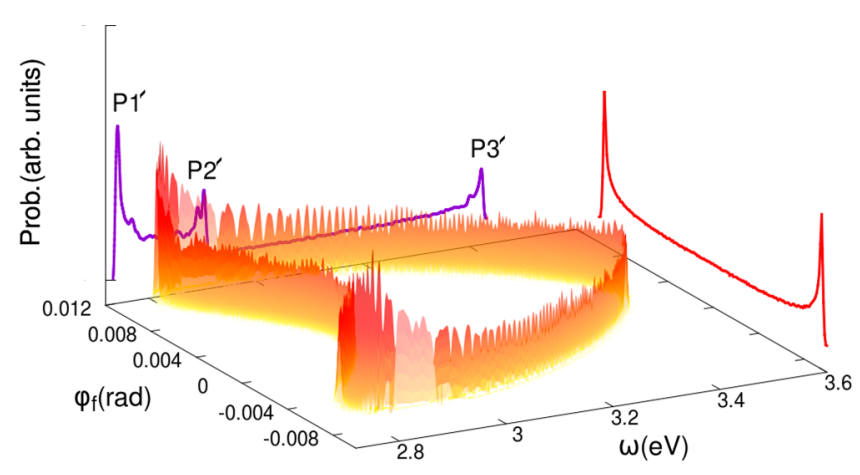

FIG. 7. (Color online) Two-dimensional angle and energy-loss distribution, as a function of the final azimuthal angle $\varphi_{f}$ and the lost energy $\omega$, for $1 \mathrm{keV}{ }^{3} \mathrm{He}$ atoms impinging on $\operatorname{Ag}(110)$ along the [1 10$]$ direction with $\theta_{i}=1^{\circ}$. Integrated angular and energy-loss distributions are also shown in the figure.

projectile momenta $\vec{K}_{f}$ almost strictly verify

$$
\varphi_{f}^{2}+\theta_{f}^{2} \simeq \theta_{i}^{2}\left(E_{i} / E_{f}\right),
$$

where $\theta_{i}$ is the incidence angle measured with respect to the surface plane. This is related to the fact that in our model the friction force acts along the direction of the velocity. Then, the energy loss affects mainly the component of the projectile momentum along the incidence channel, as proposed in Ref. [15]. In Fig. 7 we plot the double-differential probability $d P^{(\text {inel) }} / d \omega d \varphi_{f}$ for the incidence conditions of Fig. 4. This two-dimensional angle- and energy-loss-resolved distribution displays a double-peak energy-loss structure for most angles, similar to that reported in Ref. [42]. From the figure it is clear that the peak $\mathrm{P}^{\prime}$ of the energy-loss distribution is related to classical rainbow angles, which correspond to the outermost $\varphi_{f}$ angles of the spectrum. But the other two peaks- $\mathrm{P} 2{ }^{\prime}$ and $\mathrm{P} 3^{\prime}$ - come from a different angular region and they are not affected by rainbow scattering. To provide experimental confirmation of these findings, experiments in coincidence, such as the ones recently reported for the $\mathrm{He} / \mathrm{LiF}(001)$ system in Ref. [46], should be desirable.

Finally, the contribution of electronic dissipative processes to GIFAD patterns is analyzed. In Fig. 8 we plot the momentum distribution $d P / d Q_{\mathrm{tr}}$ as a function of the transverse transferred momentum $Q_{\text {tr }}=K_{f} \cos \theta_{f} \sin \varphi_{f}$ for $0.5 \mathrm{keV} \mathrm{He}$ atoms impinging along the [110] direction. Both inelastic and elastic distributions are displayed in the figure. The inelastic momentum distribution is derived from $d P^{\text {(inel) }} / d E_{f} d \Omega_{f}$ as

$$
\frac{d P^{(\text {inel })}}{d Q_{\mathrm{tr}}}=\int d E_{f} \int d \theta_{f} \frac{\cos \theta_{f}}{\sqrt{K_{f s}^{2}-Q_{\mathrm{tr}}^{2}}} \frac{d P^{\text {(inel) }}}{d E_{f} d \Omega_{f}},
$$

where $K_{f s}=K_{f} \cos \theta_{f}$ is the component of the final momentum parallel to the surface plane, while the elastic distribution $d P^{(\mathrm{el})} / d Q_{\mathrm{tr}}$ is evaluated within the SE approximation, as explained in Ref. [18], using the same DFT surface potential as for inelastic calculations. The transverse momentum distribution assuming elastic scattering displays several maxima which are placed at Bragg positions $Q_{\mathrm{tr}}=m 2 \pi / d$, with $m$ an integer number and $d$ the periodicity distance of the channel. Such an elastic pattern is produced by quantum interference 


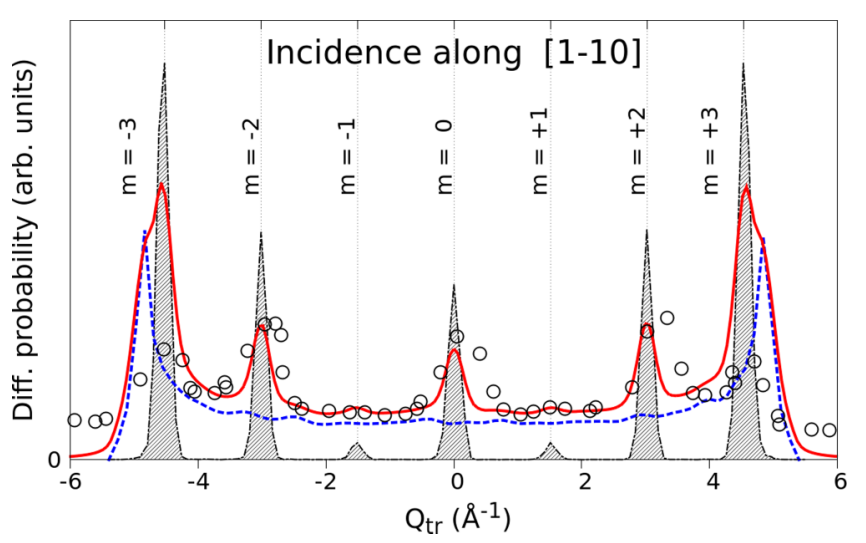

FIG. 8. (Color online) Momentum distribution, as a function of the transverse momentum transfer $Q_{\text {tr }}$, for ${ }^{3} \mathrm{He}$ atoms impinging on $\operatorname{Ag}(110)$ along the [110] direction. The incidence energy and angle are $E_{i}=0.5 \mathrm{keV}$ and $\theta_{i}=0.75^{\circ}$, respectively. Solid red line, total differential momentum probability obtained by adding elastic and inelastic contributions, as explained in the text; dashed blue line, inelastic contribution $d P^{\text {(inel) }} / d Q_{\text {tr }}$ evaluated from Eq. (3); black dashed line (shading curve), elastic contribution $d P^{(\mathrm{el})} / d Q_{\mathrm{tr}}$ evaluated within the SE approach [18]; empty circles, experimental data from Ref. [7]. The vertical dashed lines show Bragg peak positions.

of coherent transition amplitudes corresponding to different classical paths [18]. Instead, the inelastic probability is associated with different electron-hole excitations, which are here considered as incoherent processes. Then, $d P^{(\mathrm{inel})} / d Q_{\mathrm{tr}}$ presents an almost flat behavior, with only two maxima at the outermost $Q_{\text {tr }}$ values. These inelastic peaks are associated with classical rainbow scattering, as observed in Fig. 7.

In Fig. 8 we also plot differential probabilities $d P / d Q_{\mathrm{tr}}$ (red solid line) obtained by adding normalized elastic and inelastic contributions, i.e., $d P / d Q_{\mathrm{tr}}=f d P^{(\mathrm{el})} / d Q_{\mathrm{tr}}+(1-$ f) $d P^{(\mathrm{inel})} / d Q_{\mathrm{tr}}$, where $f$ is a scale factor derived by fitting the experimental data, after convoluting the sum with a Lorentz function as in Ref. [18]. For this case we found that the best fit of the experiment is obtained for $f=0.25$. Then, the relative weight of the inelastic contribution is around $75 \%$ of the total momentum distribution, higher than the one estimated in Ref. [7]. Good agreement between theoretical results and the experiment is observed in the whole range of transverse momenta, with the exception of the outermost maxima, corresponding to $m= \pm 3$, whose intensities are overestimated by the theoretical curve. This fact is related to the sharpness of the rainbow peaks, which is originated by the classical description of the projectile motion that does not include the finite intensity on the dark side of the classical rainbow [47]. However, this deficiency does not influence the momentum distribution at smaller transverse momenta. On the other hand, notice that relative intensities of the elastic peaks for $m=0, \pm 1$, and \pm 2 , which are extremely sensitive to the shape of the surface potential across the channel, remain unchanged when the inelastic probability is added, thus providing a good parameter to determine the corrugation of the surface potential.

\section{CONCLUSIONS}

We have studied the energy lost by helium atoms after grazingly colliding with a silver surface along low-indexed crystallographic directions. The distribution of inelastically scattered atoms was obtained within a semiclassical formalism that incorporates a friction force in the classical dynamics equations. This model is well suited for the calculation of the

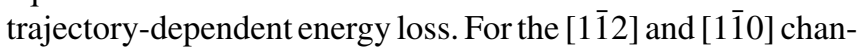
nels we found that the energy-loss distribution corresponding to a given incidence velocity displays several sharp maxima, which are related to trajectories that probe different regions of the surface interaction. These energy-loss structures might become a useful tool to study the surface electronic density corrugation. Presently, however, they completely disappear when current typical experimental spreads of the incident beam are considered [7]. Convoluted energy-loss spectra present broad distributions with only one maximum, whose position is in fairly good agreement with available experimental data $[7,16]$.

The present work suggests that the transverse momentum distribution due to inelastic electronic processes contributes to GIFAD patterns with a practically constant background, except around the outermost momentum values, where maxima related to classical rainbow scattering are present. According with our model, such an incoherent background does not affect the relative intensities of internal diffraction maxima, even though its total contribution to the momentum distribution is estimated around $75 \%$ for incidence along the [110] channel. By adding inelastic and elastic momentum distributions, the latter evaluated within the SE approximation [18], a reasonable agreement with the experimental momentum spectrum is observed. However, more work is needed to better understand the diffraction of fast atoms from metals.

\section{ACKNOWLEDGMENTS}

The authors are grateful to Philippe Roncin for a critical reading of the manuscript and for his helpful suggestions. C.R.R and M.S.G. acknowledge financial support from CONICET, UBA, and ANPCyT of Argentina. G.A.B. acknowledges financial support by ANPCyT and is also thankful to Dr. H. F. Busnengo, Dr. J. D. Führ, and Dr. M. L. Martiarena regarding the PES calculation. J.I.J. acknowledges financial support by the Basque Departamento de Educación, Universidades, e Investigación, the University of the Basque Country UPV/EHU (Grant No. IT-756-13), and the Spanish Ministerio de Ciencia e Innovación (Grant No. FIS2010-19609-C02-02).
[1] A. Schüller, S. Wethekam, and H. Winter, Phys. Rev. Lett. 98, 016103 (2007).

[2] P. Rousseau, H. Khemliche, A. G. Borisov, and P. Roncin, Phys. Rev. Lett. 98, 016104 (2007).
[3] A. Schüller and H. Winter, Phys. Rev. Lett. 100, 097602 (2008).

[4] J. R. Manson, H. Khemliche, and P. Roncin, Phys. Rev. B 78, 155408 (2008). 
[5] F. Aigner, N. Simonović, B. Solleder, L. Wirtz, and J. Burgdörfer, Phys. Rev. Lett. 101, 253201 (2008).

[6] M. S. Gravielle and J. E. Miraglia, Phys. Rev. A 78, 022901 (2008).

[7] N. Bundaleski, H. Khemliche, P. Soulisse, and P. Roncin, Phys. Rev. Lett. 101, 177601 (2008).

[8] A. Schüller, M. Busch, J. Seifert, S. Wethekam, H. Winter, and K. Gärtner, Phys. Rev. B 79, 235425 (2009).

[9] C. Díaz, P. Rivière, and F. Martín, Phys. Rev. Lett. 103, 013201 (2009).

[10] H. Winter and A. Schüller, Prog. Surf. Sci. 86, 169 (2011) and references therein.

[11] J. Seifert, M. Busch, E. Meyer, and H. Winter, Phys. Rev. Lett. 111, 137601 (2013).

[12] J. Lienemann, A. Schuller, D. Blauth, J. Seifert, S. Wethekam, M. Busch, K. Maass, and H. Winter, Phys. Rev. Lett. 106, 067602 (2011).

[13] H. Khemliche, P. Rousseau, P. Roncin, V. H. Etgens, and F. Finocchi, Appl. Phys. Lett. 95, 151901 (2009).

[14] M. Busch, A. Schüller, S. Wethekam, and H. Winter, Surf. Sci. 603, L23 (2009).

[15] H. Khemliche, N. Bundaleski, P. Soulisse, and P. Roncin, Nucl. Instrum. Methods Phys. Res. B 267, 620 (2009).

[16] N. Bundaleski, P. Soulisse, A. Momeni, H. Khemliche, and P. Roncin, Nucl. Instrum. Methods Phys. Res. B 269, 1216 (2011).

[17] M. Busch, J. Lienemann, J. Seifert, A. Schüller, and H. Winter, Vacuum 86, 1618 (2012).

[18] C. A. Rios Rubiano, G. A. Bocan, M. S. Gravielle, N. Bundaleski, H. Khemliche, and P. Roncin, Phys. Rev. A 87, 012903 (2013).

[19] A. Schüller, H. Winter, M. S. Gravielle, J. M. Pruneda, and J. E. Miraglia, Phys. Rev. A 80, 062903 (2009).

[20] M. S. Gravielle and J. E. Miraglia, Nucl. Instrum. Methods Phys. Res. B 258, 21 (2007).

[21] T. Y. Shyn, Phys. Rev. A 22, 916 (1980).

[22] J. I. Juaristi, M. Alducin, R. Díez Muiño, H. F. Busnengo, and A. Salin, Phys. Rev. Lett. 100, 116102 (2008).

[23] P. Giannozzi et al., J. Phys. Condens. Matter 21, 395502 (2009).

[24] H. F. Busnengo, A. Salin, and W. Dong, J. Chem. Phys. 112, 7641 (2000).

[25] A. Zugarramurdi and A. G. Borisov, Phys. Rev. A 86, 062903 (2012).

[26] A. Robin, W. Heiland, J. Jensen, J. I. Juaristi, and A. Arnau, Phys. Rev. A 64, 052901 (2001).
[27] R. H. Ritchie, Phys. Rev. 114, 644 (1959).

[28] J. E. Valdes, P. Vargas, C. Celedon, E. Sanchez, L. Guillemot, and V. A. Esaulov, Phys. Rev. A 78, 032902 (2008).

[29] A. Robin et al., Phys. Res. B 67, 165409 (2003).

[30] I. Goikoetxea, J. I. Juaristi, M. Alducin, and R. Díez Muiño, J. Phys.: Condens. Matter 21, 264007 (2009).

[31] L. Martin-Gondre, M. Alducin, G.A. Bocan, R. DiezMuino, and J. I. Juaristi, Phys. Rev. Lett. 108, 096101 (2012).

[32] M. Alducin, R. Díez Muiño, and J. I. Juaristi, in Dynamics of Gas-Surface Collisions, Springer Series in Surface Science, Vol. 50 (Springer, 2013), p. 371.

[33] P. M. Echenique, R. M. Nieminen, and R. H. Ritchie, Solid State Commun. 37, 779 (1981).

[34] E. Zaremba, J. H. Rose, L. M. Sander, and H. B. Shore, J. Phys. F: Met. Phys. 7, 1763 (1977).

[35] J. P. Perdew, K. Burke, and M. Ernzerhof, Phys. Rev. Lett. 77, 3865 (1996).

[36] D. Vanderbilt, Phys. Rev. B 41, 7892 (1990).

[37] N. Marzari, D. Vanderbilt, A. De Vita, and M. C. Payne, Phys. Rev. Lett. 82, 3296 (1999).

[38] B. W. Busch and T. Gustafsson, Surf. Sci. 407, 7 (1998); M. Lindroos, C. J. Barnes, and M. Valden, ibid. 218, 269 (1989); E. Holub-Krappe and K. Horn, ibid. 188, 335 (1987); Y. Kuk and L. C. Feldman, Phys. Rev. B 30, 5811 (1984).

[39] P. Roncin (private communication).

[40] Theoretical results were convoluted with a Gaussian function whose parameters were extracted from the experiment.

[41] L. Chen, J. Shen, J. E. Valdés, P. Vargas, and V. A. Esaulov, Phys. Rev. A 83, 032901 (2011).

[42] J. M. Moix, E. Pollak, and S. Miret-Artés, Phys. Rev. Lett. 104, 116103 (2010).

[43] The parameters of the convolution were extracted from Ref. [7].

[44] Note that the directions reported in Ref. [7] correspond to thermal energy atom scattering (TEAS) notations, i.e., to the direction probed by the beam rather than the direction of the beam itself, both directions being perpendicular to each other. The same notation was used for energy-loss measurements (P. Roncin, private communication).

[45] M. S. Gravielle and J. E. Miraglia, Nucl. Instrum. Methods Phys. Res. B 267, 610 (2009).

[46] J. Lienemann et al., Nucl. Instrum. Methods Phys. Res. B 315, 30 (2013).

[47] M. V. Berry and K. E. Mount, Rep. Prog. Phys. 35, 315 (1972). 